\title{
A Final Commentary: Change and Therapeutic Factors in the Short-Term Dynamic Psychotherapy with Mr. C
}

\author{
Alessandra De Coro ${ }^{1 \bowtie}$
}

In recent years there has been a growing interest in the various forms of brief psychotherapy derived from psychoanalytic principles (e.g., Alexander \& French, 1946; Mann, 1973; Strupp \& Binder, 1984), which has transformed this form of intervention into the most widely used in the field. There is now a huge amount of psychotherapeutic approaches-Kazdin alone in 1986 had already identified over 400 different psychotherapies-a number today that has certainly grown-each of which relies on its own techniques and visions of mental functioning and psychopathology. Decades of process and outcome research, however, have lead us to able to say with confidence that there is no best model of intervention but rather that all these approaches, despite their diversity, share a common therapeutic mechanism in the clinical enterprise (Norcross, 2011) and in the good functioning of the patient-therapist relationship (Lingiardi, 2002; Wampold, 2001).

The aim of this commentary is to discuss the five papers about the short-term dynamic psychotherapy of $\mathrm{Mr}$. C, in order to draw some general conclusions by crossing the results of the different studies. The task of comparing data obtained with different methodologies is not so simple, because each method is based on a specific theoretical and empirical background, even if all of them stem from the common ground of psychoanalytic and psychodynamic approaches.

In the field of psychoanalytic technique a long river of ink follows the endless and still unresolved debate about differences between psychoanalysis and psychotherapy, where the latter has taken different forms, such as "psychoanalytic psychotherapy," "dynamic psychotherapy," "supportive psychotherapy," "expressive psychotherapy" (referring to technique), or "brief

\footnotetext{
${ }^{1}$ Department of Dynamic and Clinical Psychology, Sapienza University of Rome.

$\triangle$ Correspondence concerning this article should be addressed to Alessandra De Coro, via degli Apuli, 1, 0185 Roma.E-mail: alessandra.decoro@uniroma1.it
}

psychotherapy" and "long term psychotherapy" (referring to the treatment's duration). Most frequently, however, this debate-raging since the 1940-was based on an ideological defence of "pure psychoanalysis from corruption due to clinicians' need to extend psychoanalytic theory to the understanding of severe psychiatric disorders or to the treatments in institutional settings. A small volume published in 2007 as a tribute to the vast work of Robert Wallerstein, who has been one of the major contributors to the discussion about dynamic psychotherapies (Wallerstein, 1988, 1995, 2001), is entitled From impression to inquiry (Bucci \& Freedman, 2007) and shows that, in parallel with Wallerstein's career, psychoanalytic reflexions about therapeutic change and therapeutic factors have moved more and more towards empirical research, abandoning a solipsistic tradition based on clinicians' reports and developing new methodologies to capture not only the changes obtained by patients through psychoanalysis or dynamic psychotherapies, but the very nature of the clinical exchange, that is the multiple variables which can be considered responsible for those changes.

Bucci and Freedman (2007), in their introduction to the volume, wrote:

\footnotetext{
All psychoanalytic research aims to enhance the legitimacy of our discipline. Outcome research, including effectiveness and efficacy studies, speaks to public health concerns. Process research seeks to examine what goes on in the room between analyst and patient, and what goes on within each participant. Conceptual research speaks to the needs of the psychoanalytic community in affirming the "truth value" of guiding ideas. [....] We may also talk briefly here about a vision of psychoanalytic research that looks beyond the confines of the guiding psychoanalytic concepts-questioning rather than "affirming" or "providing evidence for" them (p. 9).
}

The studies collected in this special issue, even if limited to a single case investigation, try to respond to the three aims proposed by outcome, process and conceptual inquiry. The multidimensional assessment of the treatment presented through the five papers, in fact, offers enough material in order to: 
(i) evaluate the psychotherapy effectiveness in operational terms, comparing the therapist's clinical judgement with self-report and clinician's report instruments assessing outcome;

(ii) study the verbal and non-verbal (or para-verbal) communication and interactions between patient and therapist, advancing hypotheses about what kind of macro- and micro-processes have been going on throughout the sessions and have possibly produced the effects measured;

(iii) question possible explanations of what happens in brief psychotherapies, differently from longer treatments, that may bring about kinds of therapeutic changes.

Let's just recall the characteristics of this clinical case: Mr. C, a 21-year-old university student asked for psychological help at his University's psychological Service for sexual symptoms and interpersonal difficulties, obtaining a symptomatic resolution and a better regulation of self-esteem at the end of the fourteen sessions of a brief dynamic psychotherapy (once a week, according to Davanloo's model). Further six follow-up sessions (three after six and three after sixteen months from the end of the therapy) confirmed the stability of the results, according to the therapist's qualitative evaluation and to the quantitative data obtained in the outcome study.

The positive changes after the treatment, reported as "impression" both by the patient and his therapist, have been "investigated" through both a self-report questionnaire about pathological symptoms and a clinicianreport Q-sort method for the personality assessment, which converged in the evaluation of a substantial improvement of C's clinical conditions. In terms of symptoms, the Symptom Check List-Revised (SCL-90-R: see Derogatis, 1983; Sarno, Preti, Prunas, \& Madeddu, 2011, for Italian norms), a largely used and validated instrument, shows a clear decrease from pathological values to non-pathological ones for Depression, Anxiety and Obsessive-Compulsive dimensions from Time 1 (therapy beginning: $\mathrm{T}>60=$ high intensity) to Time 2 (therapy ending: $T<50=$ some symptoms, in a mean range); these values are even still decreasing in Time 3 (sixteen months after the treatment end), indicating a stable therapeutic change, that is confirmed by the Global Score Index (GSI) of this test, which is considered the best index for the intensity of subject's psychological disorder: the GSI, in fact, decreases from $\mathrm{T}=61$ (disorder intensity from moderate to high) to $\mathrm{T}=49$ (in the mean range of non-clinical population), up to $\mathrm{T}$ $=44$ (non-relevant disorder) at the second follow up. The highest value at the last measure, Paranoid Ideation, gets $\mathrm{T}=50$ (versus $\mathrm{T}=55$ at the starting point), showing a personality trait (in the mean range of not clinical population) not influenced by psychotherapy.

As for the personality changes on the ShedlerWesten Assessment Procedure (SWAP-200; Shedler \& Westen, 2007), before the therapy C showed a Dependent personality disorder ( $T>60)$, as well as marked traits of Dysphoria and Avoidant personality $(\mathrm{T}>50)$, while at the second follow up all these scores decreased under the cut-off point, showing a reduction of dysfunctional traits. In parallel, two Q-factors dimensions, namely Depressive-high functioning and High functioning, that concern adaptive functioning and mature defences, had scores over the cut-off value since the beginning of the psychotherapy and showed a further increase at the follow up: while before they indicated the patient's good capacities of getting benefits from a psychological help, afterwards they may suggest the hypothesis that C's adaptive strategies have been reinforced by the therapeutic intervention. Remarkably, however, the Obsessive-Compulsive dimension, that can be associated to high level defence mechanisms too (see Perry, 1990), has clearly increased at the end of the therapy, growing from marked traits up to a proper Obsessive disorder $(\mathrm{T}>60)$; some obsessive characteristics of his mental functioning seem to be recognised also by the patient himself, because on the SCL-90 he still gets $\mathrm{T}=48$ at the last administration, however under the critical cut off $(T=55)$.

Some changes at the level of C's structural organization, in terms of prevailing relational schemata, can be inferred also from the final results of the Core Conflictual Relational Theme (CCRT: Luborsky \& CritsChristoph, 1998). In general, pervasiveness of the primary CCRT has not decreased from the first to the last application, but Negative Other's and Selfs Responses are considerably diminished, confirming a change towards better psychological health (see Grenyer \& Luborsky, 1998): in the last period of the therapy, in fact, both RO and RS components produce negative categories only in the secondary CCRT, while the primary one includes representations of others as "liking" him and of self as "self-controlled and self-confident," as well as "accepted and respected." It's even noticeable that these categories, which are present as first rank in the late C's therapeutic narratives, have been found to be not so much represented in late narratives of a small sample of 13 American psychotherapies: "Others like me" occupied the first rank only in $8 \%$ of cases; "I feel self controlled and self-confident" was never first, and the same for "I feel accepted and respected" (Popp et al., 1998, p. 192). Moreover, quite surprisingly for a brief psychotherapy, even the Wish category of the primary CCRT has changed in the last sessions: starting with the wish "to be loved and understood," typical of a hyper-activation of the attachment system, at the end of the therapy C expresses as first rank the wish "to be independent" and as second rank the wish "to achieve and help others," moving towards a more adult position, where autonomy, individuation and pro-social motivations acquire higher places in the hierarchy of motives of everyday life.

Comparing all these outcome data, we think that it's possible to affirm that: both at the end of his four months psychotherapy-and at the second follow-up more than one year after-Mr. $\mathrm{C}$ has obtained a clear reduction of his obsessive thoughts, as well as a resolution of his depression and anxiety symptoms; he has 
gained, moreover, some positive changes in his interpersonal difficulties, documented by the decrease of dependent and avoidant personality traits and by increased motivations towards independency, efficacy and pro-social behaviours in his relational patterns. In the last follow up sessions, however, an increase in his obsessive personality traits - up to pathological levelsuggests that the patient had to recur to neurotic defence mechanisms (such as isolation or intellectualization) in order to sustain a new way of dealing with interpersonal relationships after having left the "holding" surrounds of the therapeutic relationship.

The application of linguistic measures for the assessment of the referential process, according to Bucci's Multiple Code Theory (Bucci, 1997) and the analysis of the speech rate both for patient and therapist, together with the study of the interaction structures through Jones' Psychotherapy Process Q-set (PQS; Jones, 2000) and of the therapeutic alliance through the Collaborative Interactions Scale by Colli and Lingiardi (2009), allow to go deeper in the discussion about what happened in the course of the fourteen sessions that constitute the core of this therapeutic process. If we share Bucci's definition of therapeutic process as a "reorganization of emotion schemas that have been dissociated and distorted," the instruments applied to this single case for the process investigation are aimed to find out which kind of variables-if any - could be considered apt to promote and facilitate such a process of reorganization of previously dissociated emotion schemas. In our opinion, in fact, this kind of reorganization is a necessary condition for an appreciable change in relational patterns. In her paper in this volume, Bucci writes:

In order for change to come about, some trace of the avoided schema must be activated in the session and in the relationship; but activated in such a way that the painful affect is reduced, and the situation experienced as new, rather than as reinforcement of previous maladaptive expectations (this volume, p. 17).

Like the great majority of the patients, $\mathrm{C}$ enters into his therapy full of many negative feelings about himself and others, as different instruments show. From the most characteristic items of the first SWAP, we know that the young man "is expressing some feelings of inferiority, inadequacy and failure," "tends to blame himself or feel responsible for the negative things that happen" and "is afraid of being rejected or abandoned by people who are emotionally significant for him." In the first half of the therapy, two applications of CCRT confirm that in C's narratives of that period negative emotions colour the more frequent representations of himself in interaction with other people. Moreover, the study of macro-process through the dictionary of referential activity has brought to light significant differences between the first seven sessions and the last ones: the first half of this process has been characterized by higher expression of negative affects, by higher scores of referential activity (which, according to Multiple
Code Theory, implies the activation of symbolic function to repair the connections between emotional schemas) and by a significant co-variation of Referential Activity (RA) and reflective verbalizations. As the authors comment: "high referential activity typically belongs to the symbolization phase, while negative affects and IREF-IWRAD ${ }^{1}$ covariation index are the typical indications of the reorganization phase" (Mariani, \& De Coro, 2013, in this volume, p. 50). The correlations examined between IWRAD variables and the speech rate (a measure which calculates the speed of a speaker's talk) seem to confirm that the patient is authentically emotionally involved in his discourse: his speech slows down every time that he uses more a somatic and sensory language, as well as when the mean $\mathrm{RA}$ increases, as if he were concentrating more on his present subjective experience. Also the therapist accompanies the patient's expression of somatic and sensory references with a slower speech, as well as the patient's references to affective experience. In the study on this non-verbal dimension of verbal communication, what may be relevant to the assessment of reciprocal emotion regulation of therapeutic interactions is to note that the therapist's speech rate seems to be sensitive both to the covariation between RA and negative emotions (positive correlation) and to the covariation between positive affects and negative ones (negative correlation): it is possible to interpret these quantitative relations by hypothesizing that the therapist shows an emotional resonance (slowing his own speech) both to the vivid expression (through images) of negative affects from the patient and to the increase of explicit ambivalence (positive and negative affects increase together in the patient's discourse; see Rocco, Mariani, \& Zanelli, 2013, in this volume).

If we investigate this first half of C's therapy by analyzing his relationship with the therapist, we find that the PQS shows a change from the initial sessions up to the seventh session (taken as the central phase of the process). The first phase illustrates a prevailing interaction structure where the patient tends to be passive and his communications oscillate between self-accusatory tones ("expresses shame or guilt") and recriminations ("blames others, or external forces, for difficulties"), while the therapist is accepting him with empathic responsiveness ("is sensitive to the patient's feelings" and "is attuned to the patient"). In the second phase, both the participants are described as moving towards a more active interaction oriented towards symbolization: the therapist, still remaining "responsive and affectively involved," "draws attention to feelings regarded by the patient as unacceptable," the patient brings in the discussion "memories or reconstructions

\footnotetext{
1 The Italian Reflection Dictionary (IREF) and the Italian Weighted Referential Activity Dictionary (IWRAD) are two of the computerized dictionaries used for the linguistic measures applicable to therapist's and patient's speech. For further details, see Mariani, Rocco, \& De Coro, in this volume.
} 
of infancy and childhood," and "achieves a new understanding or insight."

It is noticeable that this Q-sort procedure, based on standardized qualitative descriptions of the interactions' contents, gives a picture of the clinical exchange very near to the abstract and quantitative descriptions based on the formal characteristics of verbal language. The IWRAD dictionaries, in fact, agree in underlining the affective attunement in verbal communication between patient and therapist through the whole process: patient's negative affects expressions appear significantly associated to therapist's negative affects and to his global affective expression, probably indicating the therapist's ability to mirror the patient's negative emotions; in turn, the crossed inverse correlation between high patient's RA and therapist's positive affects, as well as between patient's positive affects and therapist's RA, may suggest that the therapist expresses greater affective support when the patient is incapable of symbolizing, and, vice versa, tends to reduce his own emotional involvement accompanying the patient's activation of symbolic function (caught by RA higher intensity); it seems also that the therapist produces more symbolic connections where the patient's affective expressions are more silent, while he restrains his interpretive function when the patient's emotional involvement is higher. Such a representation of the clinical interactions in this therapy, provided by the linguistic measures, recalls Stern's description of the motherbaby interactive "dance," where both the participants alternate activation and quietness periods.

The data collected on the therapeutic alliance-operationally defined by the authors in terms of dynamic processes of ruptures and resolutions of the relation (Colli \& Lingiardi, 2009)-give further information about this therapeutic relationship. The collaborative level of both participants in the course of the fourteen sessions oscillates between higher and lower values, between .7 and 1.5 , with a constantly increasing trend from the first session up to the eleventh one: the patient's collaborative level is however always lower than the therapist's level, until they tend nearly to overlap in the last three sessions (see Figure 1, p. 37). In the first four sessions, the global index of therapeutic alliance increases from .9 to 1.1 , with a linear trend, and the analysis of micro-processes in the fourth session shows that in correspondence to the ruptures by the patient there are almost always some resolutions by the therapist, followed by a collaborative process by the patient (Rocco, Gennaro, De Bei, Zanelli, \& Condino, 2013, in this volume). These results allow to infer that the therapeutic alliance between $\mathrm{C}$ and his therapist-defined in a relational perspective as a complex group of unconscious and conscious mental operations that aim at maintaining a reciprocal and parithetic cooperationhas developed gradually since the first four sessions, laying the basis for a possible experience of intersubjectivity through the following process.

Session n. 7 has been called an "in-depth session" by the therapist and the researchers (Rocco et al., 2013, in this volume) and appears central in the discussion of this therapeutic process according to different instruments; from a purely clinical point of view, we can note that it is situated at the mid-point of the "journey" (as C expressed himself) and that it clearly had a longer time duration, an hour and a half, probably responding to the therapist's need to accept the separation anxiety of the patient in a moment of intense emotional involvement. This hypothesis seems to be confirmed by three different indexes: (1) the cluster analysis of the linguistic variables indicates that this seventh session is the most representative of cluster A (negative affects expression, high referential activity, clear co-variation between referential activity and reflection), which means that it is characterized by activation of emotions (specially negative ones), connections between emotions and verbal symbols, reorganization of emotional schemas; (2) the PQS shows that the interactions in this session are focused on typical therapeutic dialogue: the patient "expresses deeply felt concern," but also "achieves a new understanding or insight," and the therapist "points out patient's use of defensive manoeuvres" and "draws attention to feeling regarded by the patient as unacceptable;" (3) the micro-analysis of therapeutic alliance describes this session as different from the others because the distribution of ruptures and resolutions is unusual: the high number of ruptures by the patient, followed by resolutions by the therapist, is concentrated at the beginning, in less than one quarter of the session, while afterwards the patient becomes clearly collaborative; (4) the patient's speech rate increases in parallel with his reflective words (which may imply a distance from his immediate experience), but also with the co-variation between reflection and positive affects, while the negative correlation of the patient's speech rate with the co-variation between negative emotions and somatosensory words testify that in this session emotional schemas with a negative tone-when they are associated with immediate references to body experiencehave been evoked through a slower talking speed.

Considering all this data, we think it is possible to hypothesize that, in virtue of the increasing alliance and of the warm and attuned interactions experienced in the preceding sessions (where negative emotions had found acceptance and frequent connection to verbal meaning), just in this session $\mathrm{C}$ has activated his "avoided schema" in the therapeutic relationship, testing the therapist's ability to repair the ruptures of their relationship. Considering the patient's description with the first SWAP, he was scarcely capable of expressing his requests and his emotional needs to others: here, through ruptures and intense negative emotions, $\mathrm{C}$

\footnotetext{
${ }^{2}$ Wilma Bucci defines thus those emotional schemas which have been dissociated in a person's mind and which therefore are not easily connected to verbal meanings through the words that the patient explicitly expresses in the session (Bucci, 2013, in this volume, p. 17).
} 
places emotional pressure on the therapist. Probably, the responsiveness of the therapist (through his maintaining the alliance) and his confidence in proposing new meanings through confrontations and interpretations helped to reduce the patient's painful affects, opening a new phase in this psychotherapy, where the patient begins to experience new ways of being with another person.

In fact, in the second half of the treatment, linguistic variables are characterized by cluster B: a decrease in the dimensions of negative affects and referential activity, is associated to an increased dysfluency and a good global quantity of affects (positive + negative). That means that, while the emotional communication may go on adding the reporting of positive affects, the symbolic process seems at an end, in a long phase of consolidation of the new experience. In parallel, the therapeutic alliance, after touching the highest peak of 1.4, begins to decrease, maintaining values around 1 , but the patient's and therapist's contributions to reciprocal collaboration begin to converge in the same line of global alliance (see Figure 1 in Rocco, Gennaro, et al., in this volume, p. 37). The increase of the direct marks of rupture in the sessions from 8 to 14, together with the increasing dysfluency and the high obsessive traits in the last SWAP, suggest a partial withdrawal by $\mathrm{C}$ from the therapeutic relationship, in preparation for the final separation. The structure of the interactions revealed by the PQS in the last phase of the therapy points to a "change of direction - as the authors say (1) from a focus on symptoms to a focus on relationships; (2) from emotional issues to cognitive issues; (3) from a directive therapist to a proactive patient" (De Bei \& Montorsi, 2013, in this volume, p. 28).

The question is this: is the patient, who at the beginning of the therapy was dependent, avoidant and frequently depressively passive, now really more assertive, capable of active initiative and of asking for what he desires? Or is he now just more aware of his dysfunctional schemas and trying to convince himself and his therapist that he is independent enough not to suffer from the imminent separation? If we read the text of the last sessions, this could be a plausible formulation of our clinically founded doubts about the effectiveness of this brief psychotherapy. In my opinion, the multiple indicators that we have found and collected together through instruments that isolate different variables, created in different conceptual frames, give a systematic ground for our hypothesis about an effective change in $\mathrm{Mr}$. C at the end of his therapy, offering altogether some indications of which elements in the process had the greatest impact on this change.

These elements could be summarized as follows: cooperation in the development and maintenance of the good quality of the relationship; patient's communication of his affects, above all negative ones; construction of connections between emotions and verbal meanings; quality of reciprocal interactions and emotional involvement in the relationship. We can hypothesize that the therapeutic factors of a brief dynamic psychotherapy are not qualitatively different from those of a long-term dynamic psychotherapy, and perhaps even not so very different from the factors of a cognitive or systemic short-term psychotherapy. The specific phases of the process, however, certainly are different: in Mr. C's case, there was in the first half of the therapy an acceleration of the explorative and expressive intervention, together with a rapid increase of therapeutic alliance, which presumably created the possibility of an active transference and counter-transference experience; then both the participants gradually reduced their mutual emotional investment, but still worked together in order to reinforce the patient's coping strategies before the end of the therapy.

One last comment regards the question of the "real relationship" in psychotherapy (Gelso, 2009): is there a third component of the therapeutic relation, beyond transference and therapeutic alliance, which has to be called the real relationship to underline that what happens to a patient in psychotherapy is facilitated by the experience of a true interpersonal relationship with another person? And can what is measured by the instruments which try to enucleate contextual and dynamic aspects in the therapist-patient interactions through a systematic analysis of the transcripts of the sessions, perhaps be considered the "real relationship"? To a certain exent, I think that these scales, or Q-sort procedures, which have been standardized to capture interpersonal exchanges and their possible meanings under and beyond the here-and-now awareness of the two participants, show the complexity of the therapeutic relationship and the difficulty in distinguishing, on a phenomenological level, between the parts that dynamic clinicians have called "transference," "counter-transference" and "therapeutic alliance." In a sense, the data obtained by these instruments demonstrates that therapeutic interactions are all pertaining to a real personal relationship; but I agree with Horvath (2009) that retaining these conceptual distinctions is still useful in order to try to deepen the understanding of the complexity of the therapeutic relationship and its effectiveness for therapeutic change.

\section{References}

Bucci, W. (2013). The referential process as a common factor across treatment modalities. Research in Psychotherapy: Psychopathology, Process, and Outcome, 16(1), 16-23.

Bucci, W. (1997). Psychoanalysis and Cognitive Science. New York: Guilford Press.

Bucci, W., \& Freedman, N. (2007) (Eds.). From Impression to Inquiry. A Tribute to the Work of Robert Wallerstein. London: The International Psychoanalytical Association.

Colli, A., \& Lingiardi, V. (2009). The Collaborative Interactions Scale: A new transcript-based method for the assessment of therapeutic alliance ruptures and resolutions in psychotherapy. Psychotherapy Research, 19(6), 718-734.

De Bei, F., \& Montorsi, A. (2013). Interaction structure and transferential pattern in a brief psychotherapy. A single-case study. Research in Psychotherapy: Psychopathology, Process, and Outcome, 16(1), 24-32.

Derogatis, L. R. (1983). Administration, Scoring and Procedures 
Manual for the Revised Version. Baltimore: Clinical Psychometric Research.

Gelso, C. J. (2009). The real relationship in a postmodern world: Theoretical and empirical explorations. Psychotherapy Research, 19(3), 253-264.

Grenyer, B. F. S., \& Luborsky, L. (1998). Positive versus negative CCRT patterns. In L. Luborsky \& P. Crits-Christoph (Eds.), Understanding Transference ( $2^{\text {nd }}$ ed., pp. 55-63). Washington, DC: American Psychological Association.

Horvath, A. O. (2009). How real is the real relationship? Psychotherapy Research, 19(3), 273-277.

Jones, E. E. (2000). Therapeutic Action: A Guide to Psychoanalytic Therapy. Northvale, NJ: Jason Aronson.

Luborsky, L., \& Crits-Christhoph, P. (Eds.). Understanding Transference ( $2^{\text {nd }}$ ed.). Washington DC: American Psychological Association.

Mariani, R., \& De Coro, A. (2013). Study of a short-term treatment by referential activity linguistic measures. Research in Psychotherapy: Psychopathology, Process, and Outcome, $16(1), 42-53$

Perry, J. C. (1990). Defense Mechanism Rating Scale (5 $5^{\text {th }}$ ed.). Cambridge Hospital, Harvard Medical School, Boston.

Popp, C., Diguer, L., Luborsky, L., Faude, J., Johnson, S., Morris, M., Schaffer, N., Schaffer, P., \& Schmidt, K. (1998). The parallel of the CCRT from waking narratives with the CCRT from dreams: a further validation. In L. Luborsky \& P. Crits-Christoph (Eds.), Understanding Transference $\left(2^{\text {nd }}\right.$ ed, pp. 187-196). Washington, DC: American Psychological Association.

Rocco, D., Gennaro, A., De Bei, F., Zanelli, D., \& Condino, V. (2013). Development and characteristics of the therapeutic alliance in a short term dynamic psychotherapy: a descriptive case study. Research in Psychotherapy: Psychopathology, Process, and Outcome, 16(1), 33-41.

Rocco, D., Mariani, R., Zanelli, D. (2013). The role of non-verbal interaction in a short term psychotherapy: preliminary analysis and assessment of paralinguistic aspects. Research in Psychotherapy: Psychopathology, Process, and Outcome, 16(1), 53-64.

Sarno, I., Preti, E., Prunas, A., \& Madeddu, F. (2011). SCL-90$R$ : Symptom Checklist 90-R. Adattamento italiano. Firenze: Giunti O. S.

Shedler, J., \& Westen, D. (2007). The Shedler-Westen Assessment Procedure (SWAP): Making Personality Diagnosis Clinically Meaningful. Journal of Personality Assessment, 89(1), 41-55.

Wallerstein, R. S. (1988). Assessment of structural change in psychoanalytic therapy and research. Journal of the American Psychoanalytic Association (Suppl.), 36, 241-261.

Wallerstein, R. S. (1995). The talking cures: the psychoanalyses and the psychotherapies. New Haven CT: Yale University Press.

Wallerstein, R. S. (2001). The generations of psychotherapy research: An overview. Psychoanalytic Psychology, 18, 243-267.

Received February 5, 2013

Revision received April 12, 2013

Accepted June 24, 2013 